\title{
QUAL O SEU DIAGNÓSTICO?
}

Luciano Lago de Souza Franco ${ }^{1}$, Cresio Flores Rolim ${ }^{1}$, Regiany Alessandra Garcia ${ }^{2}$, Edivaldo Massazo Utiyama $^{3}$, Cláudio Birolini ${ }^{3}$, Cristina A.T.S. Mitteldorf ${ }^{4}$, Flávio Albertotti ${ }^{5}$, Marcos Roberto de Menezes $^{5}$, Cézar José Albertotti ${ }^{6}$, Giovanni Guido Cerri ${ }^{7}$

Trabalho realizado no Hospital Sírio Libanês, São Paulo, SP. 1. Médicos Residentes do Hospital Sírio Libanês. 2. Médica Preceptora da Residência de Radiologia do Hospital Sírio Libanês. 3. Médicos Cirurgiões do Corpo Clínico do Hospital Sírio Libanês. 4. Médica Patologista Assistente do Laboratório de Patologia Cirúrgica e Molecular do Hospital Sírio Libanês. 5. Médicos Assistentes do Serviço de Diagnóstico por Imagem do Hospital Sírio Libanês. 6. Médico Responsável pelo Serviço de Tomografia Computadorizada do Hospital Sírio Libanês. 7. Professor Titular de Radiologia da Faculdade de Medicina da Universidade de São Paulo, Coordenador do Centro de Diagnóstico do Hospital Sírio Libanês. Correspondência: Dr. Luciano Lago de Souza Franco. E-mail: lucianolsfranco@yahoo.com.br

Paciente do sexo feminino, 35 anos de idade, com antecedentes de fibromialgia e estenose da junção pielo-ureteral à direita, apresentando dor intensa do tipo cólica na fossa ilíaca direita, com inicio há três dias, junto com o período menstrual.
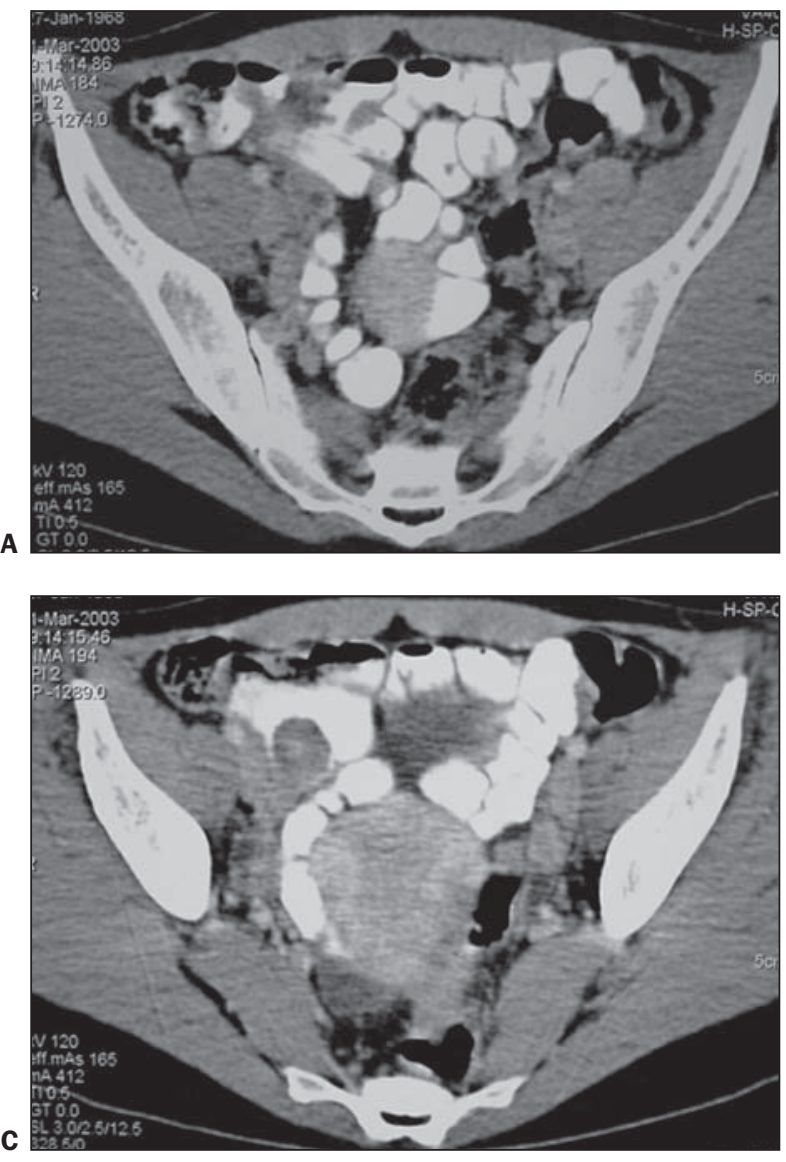

Figura 1(A,B,C,D). Tomografia computadorizada.

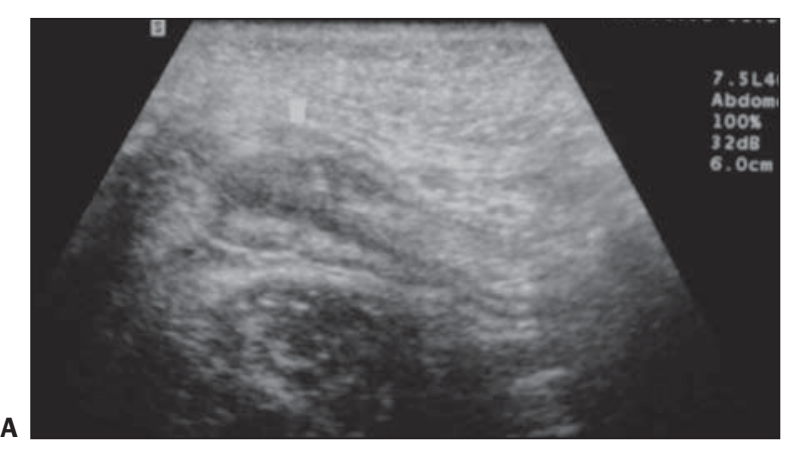

Figura 2(A,B). Ultra-som.
Negava febre, náuseas, vômitos ou alteração do hábito intestinal. Ao exame físico, encontrava-se eutrófica, em bom estado geral, com abdome plano, flácido, doloroso à palpação e com sinais de irritação peritoneal na fossa ilíaca direita.
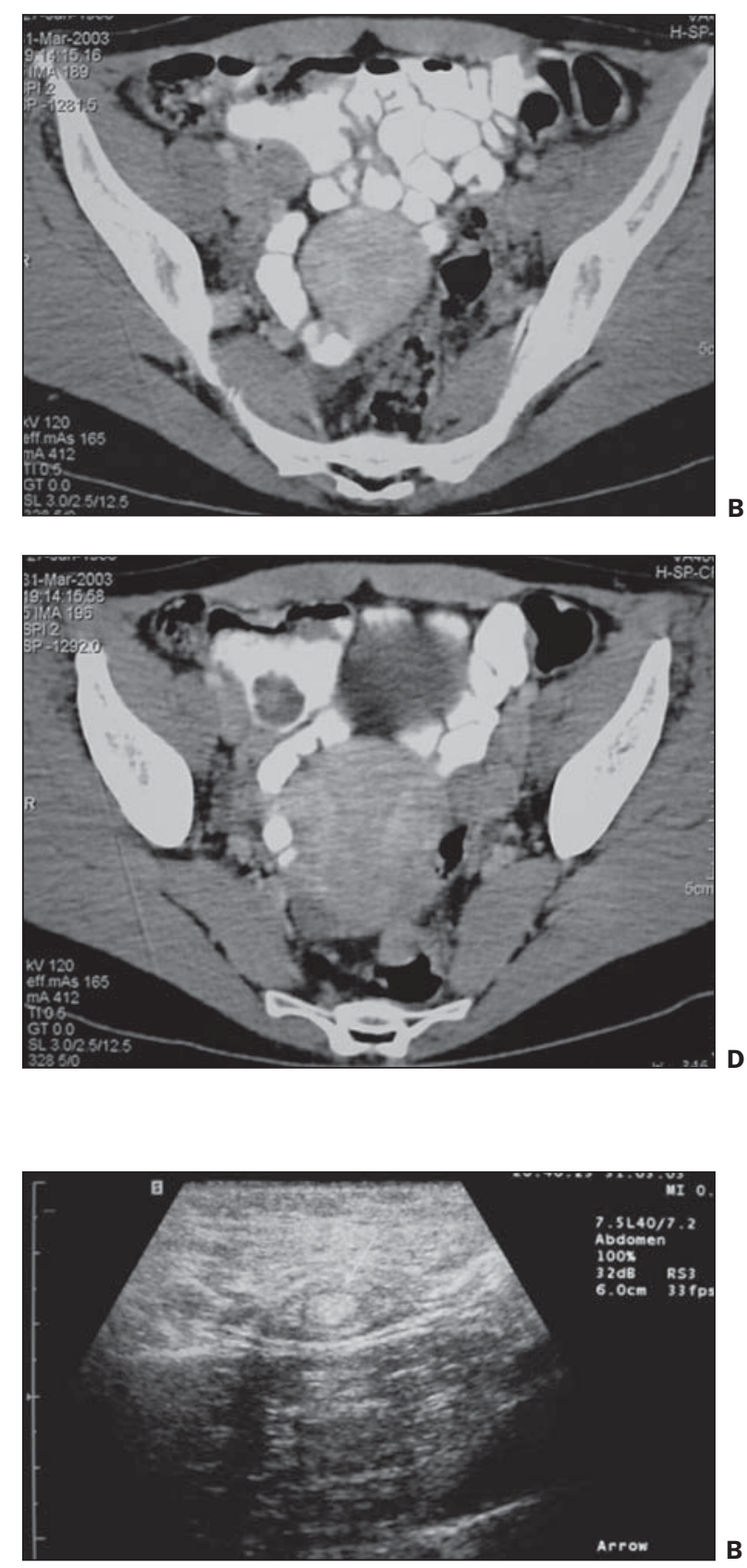
Diagnóstico: Apendicite em apêndice invaginado por endometriose.

\section{Achados de imagem}

Foram realizados estudos com ultra-sonografia e tomografia computadorizada.

Na ultra-sonografia nota-se imagem tubuliforme insinuando-se para o interior do ceco, que apresenta paredes espessadas.

A tomografia computadorizada mostra imagem tubuliforme junto ao ceco, que se insinua para o seu interior, estando a porção intracecal totalmente circunscrita pelo contraste administrado por via oral. A imagem não corresponde ao íleo terminal/ válvula íleo-cecal, que também foi individualizado.

\section{COMENTÁRIOS}

Intussuscepção do apêndice é uma alteração $\operatorname{rara}^{(\mathbf{1 - 7})}$, e foi descrita primeira- mente por $\mathrm{McKidd}^{(\mathbf{8})}$ em 1858. Ocorre em de cerca de $0,01 \%$ da população ${ }^{(9)}$ e pode surgir em qualquer grupo etário, mas aproximadamente metade dos casos se desenvolve nos primeiros 15 anos de vida ${ }^{(2)}$. Tende a ocorrer em uma freqüência cinco vezes maior no sexo masculino ${ }^{(\mathbf{3})}$.

A maior parte dos pacientes é sintomática, embora exemplos de casos assintomáticos já tenham sido registrados. Os sintomas descritos são variados e inespecíficos. Classicamente, sua sintomatologia pode ser dividida em quatro grupos $\left.{ }^{(2-7)}: 1\right)$ casos simulando apendicite aguda; 2) casos apresentando sintomas de intussuscepção intestinal - dor abdominal e vômitos com duração de vários dias, algumas vezes associados a constipação, diarréia ou melena; 3) casos com ataques recorrentes de dor abdominal intensa no quadrante inferior direito, geralmente com vômitos e melena; 4) casos assintomáticos.
A patogênese da intussuscepção do apêndice pode ser anatômica ou patológi$\mathrm{ca}^{(2-6)}$. As causas anatômicas são: a) apêndice completamente móvel, sem fixação pelas pregas peritoneais congênitas ou adesões inflamatórias; b) parede apendicular móvel, capaz de apresentar peristalse ativa; c) mesoapêndice delgado, livre de gordura e com base estreita; d) lúmen apendicular largo, com o lúmen proximal de diâmetro maior que o da porção distal; e) ceco do tipo fetal (em formato de funil). As causas patológicas são: a) corpo estranho - fecalitos ou parasitas; b) inflamação - endometriose ou hiperplasia folicular linfóide; c) neoplasia - tumor carcinóide, carcinoma, mucocele, pólipo, papiloma, fibroma, lipoma, cistos ou adenocarcinoma cecal; d) invaginação do coto apendiceal após apendicectomia.

Embora a endometriose do apêndice já tenha sido bem descrita, sua associação
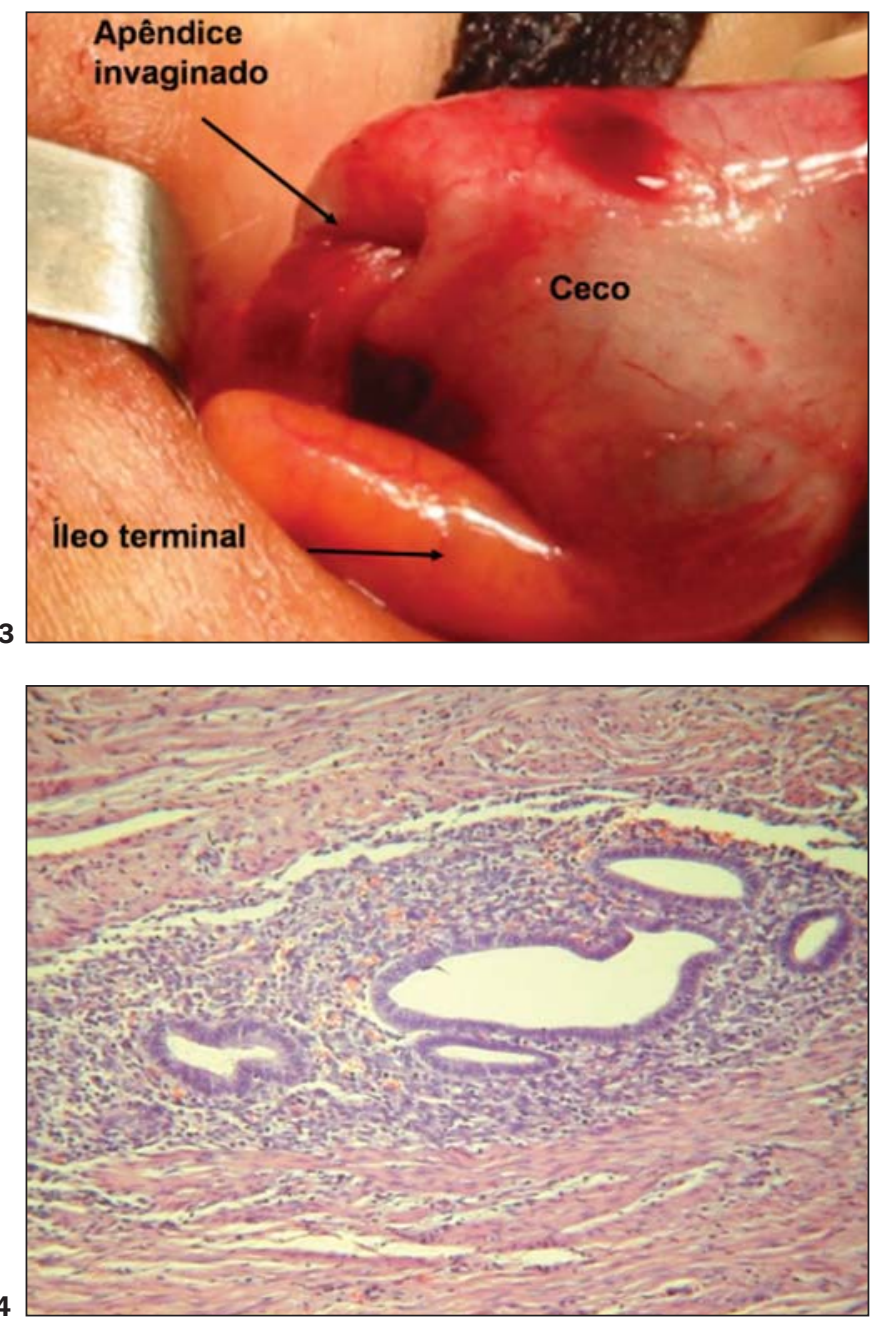

Figura 3. Imagem intra-operatória do apêndice invaginado.

Figura 4. Corte histológico do apêndice cecal demonstrando foco de endometriose na camada muscular própria, identificando-se o componente glandular e o estromal.

Figura 5. Corte em médio aumento da mucosa, que apresenta erosão do epitélio superficial de revestimento, focos de hemorragia intersticial recente e exuberante componente neutrofílico na lâmina própria, caracterizando apendicite aguda.

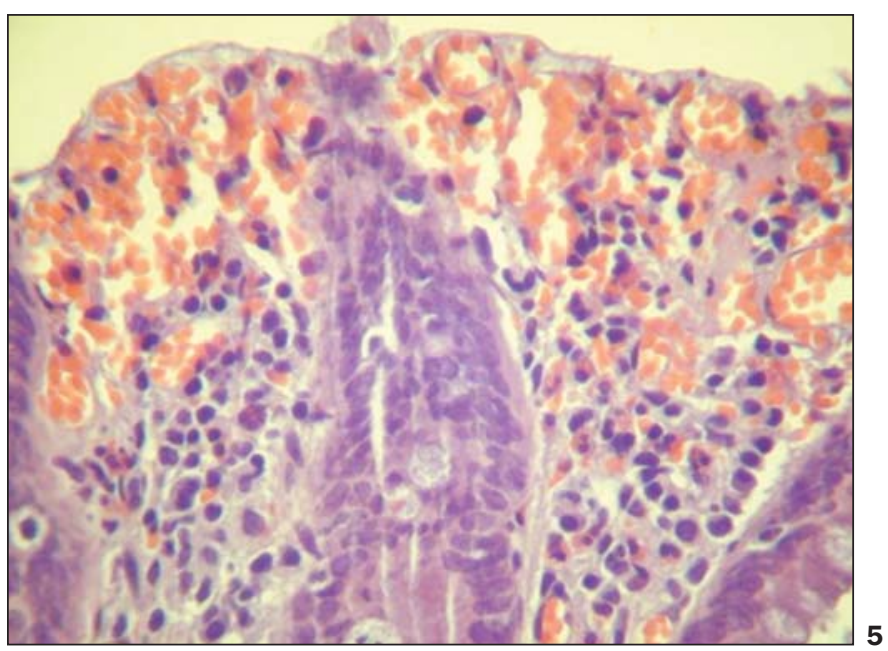


com intussuscepção do apêndice é distintamente rara, com somente 15 casos descritos na literatura ${ }^{(3)}$. A apendicite aguda, por sua vez, pode ser produzida pela retenção de material infectado na extremidade do apêndice pela obstrução do seu lúmen proximal provocada pela intussuscepção ${ }^{(2)}$.

O tratamento, neste caso, é cirúrgico. Quando a intussuscepção é parcial ou é possível sua redução pela combinação de tração e pressão, uma apendicectomia tradicional pode ser realizada. Quando a redução é impossível ou o apêndice está totalmente invertido, uma ressecção parcial do ceco é necessária ${ }^{(\mathbf{2}, \mathbf{4 , 5}, \mathbf{7})}$.

A intussuscepção do apêndice é considerada entidade de difícil diagnóstico radiológico $^{(11)}$. Várias modalidades por imagem podem demonstrar o aspecto clássico de "casca de cebola", produzido pelo encaixe do intussuscepto sobre o intussuscepiente. No início, a intussuscepção lembra uma massa em forma de alvo, associada à obstrução. À medida que a parede do intussuscipiente ao redor torna-se mais edemaciada, maior espessamento pode ser visto. Ao final, uma massa amorfa associada a obstrução grave pode se desenvolver, levando a necrose intestinal ${ }^{(\mathbf{1 2})}$.
No enema com bário, a ausência de contrastação apendiceal, juntamente com a falha de enchimento na localização esperada do apêndice, é altamente sugestiva, embora não totalmente específica, para esta entidade. No enema com duplo-contraste pode se apresentar com o aspecto em "casca de cebola" na localização esperada do apêndice, que também não demonstra contrastação do seu lúmen ${ }^{(\mathbf{1 3})}$. No exame ultra-sonográfico, pode produzir um aspecto "em forma de alvo" ou de "sinal dos anéis concêntricos" (13).

A tomografia computadorizada tem sido defendida como o método de imagem mais utilizado e acurado para o diagnóstico de intussuscepção ${ }^{(\mathbf{1 2})}$. Demonstra área central de baixa atenuação envolvida por uma camada de padrão estratificado, produzida pelas listras intercaladas de baixa e alta atenuação. Os achados podem também sugerir a causa precipitante, de importância particular em adultos, em que os processos malignos representam $20 \%-50 \%$ das causas desencadeantes ${ }^{(\mathbf{1 3})}$.

\section{REFERÊNCIAS}

1. Takase M, Sumiyama Y, Watanabe M, Abe T. A case of appendiceal endometriosis with intussusception. Jpn J Gastroenterol Surg 2001;34:1457-60.
2. Genell S. Intussusception of the appendix caused by an adenovillous papilloma. Ann Chir Gynaecol 1976;65:187-90.

3. Jevon GP, Daya D, Qizilbash AH. Intussusception of the appendix: a report of four cases and review of the literature. Arch Pathol Lab Med 1992;116: 960-4.

4. Lauwers GY, Prendergast NC, Wahl SJ, Bagchi S. Invagination of vermiform appendix. Dig Dis Sci 1993;38:565-8.

5. Geerdsen J, Axelsson C, Weile F. Invagination of the vermiform appendix: a report of two cases associated with endometriosis. Acta Chir Scand 1976; 142:417-9.

6. Nycum LR, Moss H, Adams JQ, Macri CI. Asymptomatic intussusception of the appendix due to endometriosis. South Med J 1999;92:524-5.

7. Panzer S, Pitt HA, Wallach EE, Thuluvath PJ. Intussusception of the appendix due to endometriosis. Am J Gastroenterol 1995;90:1892-3.

8. McKidd J. Case of invagination of cecum and appendix. Edinburgh Med J 1858;4:793.

9. Collins DC. Seventy-one thousand humans appendix specimens: a final report summarizing forty years study. Am J Proctol 1963;14:365-81.

10. McSwain B. Intussusception of the appendix. South Med J 1941;34:263-71.

11. Levine MS, Trenkner SW, Herlinger H, Mishkin JD Reynolds JC. Coiled-spring sign of appendiceal intussusception. Radiology 1985;155:41-4.

12. Ravin CE, Bergin D, Bisset GS III, et al. Image interpretation session: 2000. RadioGraphics 2001; 21:267-87.

13. Warshauer DM, Lee JK. Adult intussusception detected at CT and MR imaging: clinical-imaging correlation. Radiology 1999;212:853-60. 